$\overline{A E E T}$

ASOCIACIÓN ESPAÑOLA DE ECOLOGÍA TERRESTRE

\title{
Efecto de la gestión para las especies de caza menor sobre la fauna no cinegética
}

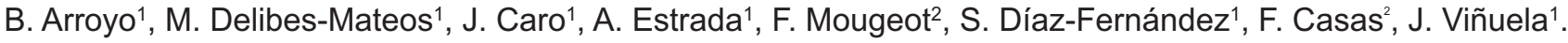 \\ (1) Instituto de Investigación en Recursos Cinegéticos, IREC (CSIC-UCLM-JCCM), Ronda de Toledo s/n, 13005 Ciudad Real, Spain \\ (2) Estación Experimental de Zonas Áridas (EEZA-CSIC), Carretera de Sacramento, La Cañada de San urbano, 04120 Almería, Spain
}

${ }^{*}$ Autor de correpondencia: B. Arroyo [beatriz.arroyo@uclm.es]

> Recibido el 8 de febrero de 2013, aceptado el 27 de mayo de 2013

\begin{abstract}
Arroyo, B., Delibes-Mateos, M., Caro, J., Estrada, A., Mougeot, F., Díaz-Fernández, S., Casas, F., Viñuela, J. (2013). Efecto de la gestión para las especies de caza menor sobre la fauna no cinegética. Ecosistemas 22(2):27-32. Doi.: 10.7818/ECOS.2013.22-2.05

La caza, como actividad que se ejerce en el medio natural y por la gestión que la acompaña, puede tener efectos en el medio ambiente. Las herramientas de gestión de caza menor más frecuentes incluyen el manejo de hábitat, el aporte de agua o comida, el control de depredadores, o la suelta de animales criados en granja. La información revisada muestra que el mantenimiento o creación de hábitats adecuados para las especies cinegéticas (vegetación natural en la matriz agrícola, paisaje en mosaico o linderos), así como el aporte suplementario de agua y comida, parecen favorecer a otras especies. El control legal de depredadores parece ser beneficioso para ciertas presas no cinegéticas, aunque este efecto no se observa en todas las especies estudiadas. Además, se usan con frecuencia métodos de control ilegales con efectos muy negativos en depredadores protegidos. La gestión demasiado intensiva, sobre todo cuando está basada en la suelta de animales de granja, parece perjudicar a determinadas especies de aves. Las molestias asociadas a la caza (ruido, presencia de humanos o perros) también perjudican a ciertas aves no cinegéticas. Estos resultados sugieren que una gestión que combine el mantenimiento de hábitats adecuados, un uso apropiado de bebederos y comederos, una caza basada en poblaciones naturales sostenibles (sin suelta de animales criados en granja), la regulación de la presión de caza, el mantenimiento de zonas de reserva, y en algunos casos un control de depredadores legal y selectivo, podría beneficiar a otras especies de interés para la conservación.
\end{abstract}

Palabras clave: Caza, Conflictos, Conservación, Herramientas de gestión, Paisajes multifuncionales

Arroyo, B., Delibes-Mateos, M., Caro, J., Estrada, A., Mougeot, F., Díaz-Fernández, S., Casas, F., Viñuela, J. (2013). Effect of small game management on non-game fauna. Ecosistemas 22(2):27-32. Doi.: 10.7818/ECOS.2013.22-2.05

Hunting, as an activity implemented over wildlife, and because of its associated management, can have effects on other species. Most frequently used management tools for small game include habitat modifications, provision of supplementary food or water, predator control or release of farmreared animals. Information reviewed shows that maintaining appropriate habitats for game animals (a heterogeneous landscape with natural vegetation patches within the farmland matrix, vegetation along field edges) and provision of supplementary food and water frequently benefit non-game species. Predator control could be beneficial for certain non-game prey, but this effect is not observed in all studied species. In addition, this tool is associated with the use of illegal methods with very detrimental effects on protected predators. Very intensive management, particularly when based on releases of farm-reared animals, is apparently detrimental for certain species. Disturbance associated with hunting activities also appears to have negative effects on some non-game species. These results show that management combining conservation of adequate habitats, an appropriate use of feeders and water points, hunting based on wild populations (without releases of farm-reared animals), regulating hunting pressure, maintaining hunt-free reserves within the hunting estates, and sometimes the use of a legal and selective predator control, could benefit other species of conservation concern.

Key words: Conflicts, Conservation, Hunting, Management tools, Multifunctional landscapes

\section{Introducción}

La caza es una forma de explotación de la fauna como recurso natural renovable muy extendida en el mundo, bien como fuente de alimento (sobre todo tradicionalmente, o en países menos desarrollados), bien como actividad de ocio. Hay evidencias sobre la capacidad de esta actividad para diezmar o incluso extinguir especies (Halliday 1980), y también sobre que los intereses cinegéticos se traducen a veces en esfuerzos de conservación (Oldfield et al. 2003, Tapper 2005). Esto convierte la caza en una actividad importante en el contexto de biología de la conservación. En general, la relación entre cazadores y conservacionistas es conflictiva, debido a la distinta percepción que tienen ambos colectivos de los costes o beneficios que la caza tiene en la conservación de los ecosistemas y su biodiversidad.

En España esta actividad la practican casi un millón de personas y atrae a más de 70000 cazadores extranjeros cada año (FACE 2005, Reginfo 2008). Además, el alcance geográfico de esta actividad es considerable: sólo los terrenos de caza gestionados de forma privada (conocidos como "cotos de caza" o "cotos cinegéticos") ocupan el $75 \%$ de la superficie nacional (MARM 2006). La caza menor es particularmente importante en España, y se practica en la mayor parte del territorio nacional (MARM 2006, RíosSaldaña 2010). Entre las especies de caza menor, las más 
relevantes (tanto a nivel numérico como socio-económico) son el conejo (Oryctolagus cuniculus) y la perdiz roja (Alectoris rufa). En las últimas décadas, las poblaciones de estas dos especies han disminuido notablemente (Delibes-Mateos et al. 2009, BlancoAguiar et al. 2012), mientras que el interés económico por su caza sigue siendo alto.

Como en otros países europeos, en la mayoría de los cotos españoles dedicados a la caza menor se utilizan una serie de herramientas de gestión destinadas a aumentar o mantener las poblaciones cinegéticas. Las medidas de gestión más comunes en aquellos cotos dedicados fundamentalmente a perdices o conejos incluyen la gestión del hábitat, el aporte de agua o comida, el control de depredadores o la suelta de animales criados en granja (Arroyo et al. 2012). Además, la gestión cinegética incluye la regulación de la presión de caza, bien numéricamente (controlando los cupos de piezas abatidas, el horario de la jornada cinegética, o el número de días de caza), bien espacialmente (por ejemplo, manteniendo zonas de reserva en las que no se puede cazar) (Angulo y Villafuerte 2003).

En este trabajo revisamos la información existente sobre el efecto que las medidas anteriores puedan tener en la fauna no cinegética. Nos centramos principalmente en los estudios más relevantes en España y el resto de Europa, con el objetivo de facilitar un debate constructivo sobre la relación entre gestión cinegética y conservación de la biodiversidad.

\section{Efectos de la gestión y conservación del hábitat}

El manejo de hábitats es una herramienta de gestión cinegética relativamente frecuente (v.g. Ferreira et al. 2013), que trata de aumentar la disponibilidad de alimento para las especies de caza, por ejemplo mediante la siembra de cereal y pastos, o mediante la ampliación y conservación de zonas seguras de cría donde las especies cinegéticas puedan encontrar refugio a salvo de depredadores. Algunas de estas prácticas también afectan a las especies no cinegéticas, sobre todo en los paisajes agrícolas.

En ecosistemas agrícolas, el uso de herbicidas y pesticidas, junto con la destrucción por roturación de los márgenes, linderos y parches de vegetación natural, como resultado de la intensificación agraria, están poniendo en peligro la biodiversidad de estos paisajes (Donald et al. 2006). Sin embargo, el manejo de ciertos hábitats para la caza menor podría ser un factor importante para la conservación de especies propias de estos medios. Por ejemplo, diferentes trabajos realizados en el Reino Unido han encontrado mayores abundancias de aves o invertebrados en cultivos sembrados para las especies cinegéticas que en los cultivos convencionales (Stoate et al. 2003, Henderson et al. 2004, Parish y Sotherton 2004, Sage et al. 2005)

El conocimiento del efecto de estas prácticas sobre la fauna no cinegética en la región Mediterránea, donde la agricultura es más extensiva, ha recibido hasta ahora poca atención, a pesar del valor para la conservación de esta zona y de la importancia socioeconómica que tiene la caza en esta región (Myers et al. 2000, Martínez et al. 2002). No obstante, la información existente sugiere que algunas asociaciones positivas como las mencionadas, también se encuentran en estas latitudes. En España, la perdiz roja y el conejo alcanzan grandes densidades en medios agrícolas heterogéneos, los denominados "paisajes en mosaico", que intercalan diferentes cultivos y parcelas no cultivadas (barbechos vegetados, labrados y rastrojos) con pequeñas manchas de vegetación natural y presencia de linderos con vegetación natural (Fig.1) (Angulo 2003, Buenestado et al. 2009, Casas y Viñuela 2010). Algunos gestores cinegéticos intentan fomentar la presencia de estas características en sus cotos de caza, que también benefician indirectamente a otras especies: por ejemplo, los linderos favorecen una mayor abundancia y diversidad de aves (Conover et al. 2007) e invertebrados (Hassall et al. 1992, Smith et al. 2008). Globalmente, el paisaje en mosaico, la presencia de vegetación natural dentro de la matriz agrícola y la presencia de lin-

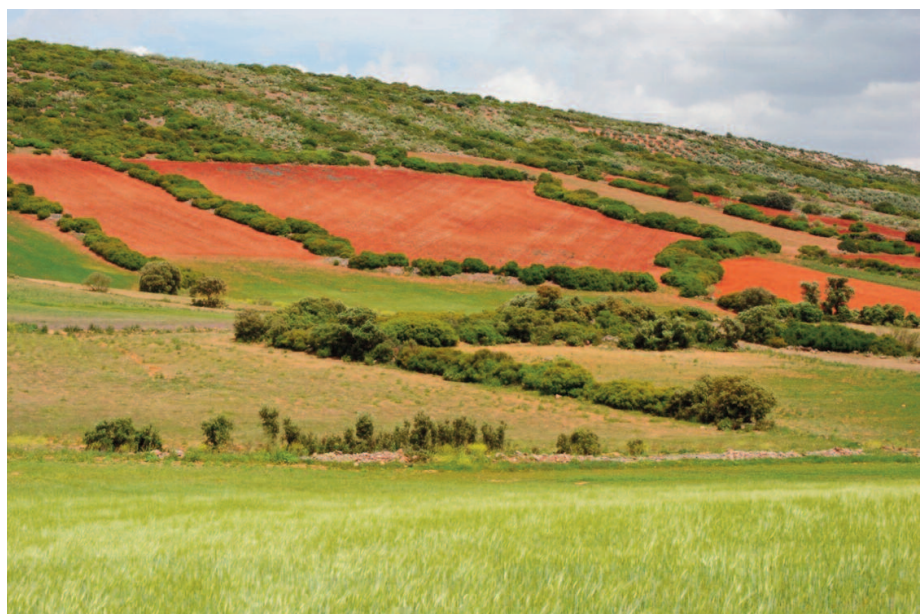

Figura 1. Mantener lindes entre cultivos y manchas de vegetación natural no sólo es esencial para obtener elevadas densidades de caza menor, sino también para aumentar la diversidad y abundancia de especies no cinegéticas en medios agrícolas. Típico paisaje en mosaico de un coto de caza de Castilla La-Mancha (España).

deros se consideran indicadores de Zonas Agrarias de Alto Valor Natural (ZAVN; Olivero et al. 2011), consideradas como indicadores de alta biodiversidad de fauna agrícola, y a promover dentro de las nuevas políticas agrarias de la Unión Europea. Es decir, la gestión de hábitats adecuados para la perdiz y el conejo puede beneficiar potencialmente a muchas otras especies en medios agrícolas. Queda por ver, no obstante, hasta qué punto es factible llevar a cabo esta medida de gestión y modificar el paisaje en casos en los que la propiedad del suelo es distinta de la titularidad de los derechos cinegéticos, como sucede en la mayoría de los cotos españoles (Arroyo et al. 2012).

Varios estudios indican, no obstante, que la actividad cinegética ha permitido en algunos casos la conservación a escala de paisaje de hábitats que son beneficiosos para otras especies. Por ejemplo, en Escocia la caza del lagópodo (Lagopus I. scoticus) ha facilitado la conservación de brezales de montaña, un hábitat considerado prioritario por las especies de interés de conservación que lo utilizan (Robertson et al. 2001). Los intereses cinegéticos para la caza de faisanes (Phasianus colchicus) han facilitado también el mantenimiento de zonas boscosas intercaladas con las parcelas agrícolas que favorecen a otras especies (Robertson 1992, Hinsley y Bellamy 2000). En el caso de la Península Ibérica, se ha observado que los cotos de caza comerciales tienen en promedio mayores superficies de hábitats naturales o semi-naturales (incluyendo dehesas, monte mediterráneo o pastizales) que los cotos que no se explotan económicamente (Arroyo et al. 2012), lo cual podría favorecer a determinadas especies típicas del monte mediterráneo. Por ejemplo, estos paisajes heterogéneos son importantes para especies emblemáticas como el lince lbérico (Lynx pardinus) (Palomares et al. 2001) o los preadultos en dispersión de águila perdicera (Aquila fasciata) y águila real (Aquila chrysaetos) (Caro et al. 2010).

Otro aspecto de la gestión del hábitat que puede favorecer a otras especies es el aporte directo de comida y agua para las especies cinegéticas. Por ejemplo, el éxito reproductor y la supervivencia invernal de varias especies de paseriformes en Gran Bretaña aumentaban con el aporte de grano para los faisanes (Stoate y Szczur 2001). En el norte de España, Gaudioso-Lacasa y colaboradores (2010) observaron que las especies no cinegéticas también usaban los bebederos diseñados para las perdices. Más recientemente, otro estudio ha encontrado que la abundancia de aves esteparias era mayor en cotos con una mayor densidad de comederos y bebederos (Estrada et al. datos sin publicar), siendo este efecto más importante en el caso de especies granívoras como la ganga ibérica (Pterocles alchata) y la ganga ortega (P. orientalis). 


\section{Efectos del control de depredadores}

En general, los cazadores y gestores cinegéticos consideran que los depredadores limitan las poblaciones de especies de caza menor y que el control de estas especies es indispensable para el mantenimiento de la actividad cinegética (Delibes-Mateos et al. 2013). Por ello, el control de depredadores es una de las prácticas de gestión más extendidas en España, así como en otros países europeos (Arroyo y Beja 2002), aunque no haya evidencia científica de qué depredadores actúan como limitantes de las especies de caza menor (Valkama et al. 2005). Aunque el control de depredadores debe restringirse a determinadas especies generalistas, la realidad es que también afecta a depredadores protegidos por la ley. Por ejemplo, casi la mitad de las muertes no naturales de águilas imperiales ibéricas (Aquila adalberti) registradas en España en el periodo 1989-2004 estaban asociadas con el control de depredadores (González et al. 2007). De igual modo, las principales causas de mortalidad del lince ibérico durante las últimas décadas han sido el trampeo y los disparos llevados a cabo por cazadores (Rodríguez y Delibes 2004). El control de depredadores afecta a especies protegidas bien porque los métodos empleados legalmente no son selectivos, como lazos o cajas trampa, o porque se llevan a cabo prácticas ilegales (Delibes-Mateos et al. 2013). Además de cepos y lazos sin freno es particularmente preocupante el uso ilegal de venenos, un método no selectivo que afecta peligrosamente a muchas especies (Viñuela y Villafuerte 2003, Whitfield et al. 2003, Mateo-Tomás et al. 2012, Márquez et al. 2013).

En numerosos casos se ha demostrado que la presencia o abundancia de depredadores protegidos es menor de lo esperado en zonas donde la gestión cinegética es más intensa o en zonas de conflicto con intereses cinegéticos (Etheridge et al. 1997, Villafuerte et al. 1998, Whitfield et al. 2004, Anderson et al. 2009, Beja et al. 2009, Amar et al. 2012, Smart et al. 2010). En el centro de España, Virgós y Travaini (2005) observaron que la riqueza de carnívoros era menor en cotos de caza gestionados intensivamente. Igualmente, Villafuerte y colaboradores (1998) sugirieron que la llegada de la enfermedad hemorrágica del conejo a España posiblemente provocó un incremento del control de depredadores en cotos de caza, lo que pudo afectar a la distribución de depredadores protegidos como el milano real (Milvus milvus). Por otra parte, en Portugal se encontró una relación negativa entre la abundancia relativa de ciertas especies de rapaces y el número de guardas de los cotos de caza (Beja et al. 2009), lo que sugeriría de nuevo que el control de depredadores puede afectar negativamente a ciertas especies protegidas. Independientemente de los distintos puntos de vista éticos sobre el control de depredadores (Leopold y Chamberlain 2002), éste sigue siendo un punto crucial de conflicto entre los cazadores y otros grupos sociales, y uno de los temas que deberían resolverse más rápidamente para permitir una mejor colaboración entre los cazadores y otros grupos conservacionistas.

Por otro lado, se ha visto que en algunos casos los depredadores protegidos son más abundantes en cotos de caza. Por ejemplo, Delibes-Mateos y colaboradores (2007) encontraron que las rapaces eran más abundantes y su comunidad más diversa en aquellas zonas donde había muchos conejos (Delibes-Mateos et al. 2007), siendo gran parte de ellas cotos de caza (Delibes-Mateos et al. 2009). A nivel de especie también se ha observado una correlación positiva entre la abundancia del águila imperial ibérica y la de su presa principal, el conejo (Delibes-Mateos et al. 2007). Resultados similares se obtuvieron en Portugal en el caso del busardo ratonero (Buteo buteo) (Beja et al. 2009). Esto se debe a que las medidas de gestión empleadas por los cazadores favorecen la presencia y abundancia de las especies cinegéticas (Delibes-Mateos et al. 2008a, Beja et al. 2009, Díaz-Fernández et al. 2013), que son el componente principal de la dieta de numerosos depredadores de gran interés para la conservación (p. ej. Yanes et al. 1998, Valkama et al. 2005, Delibes-Mateos y Gálvez-Bravo 2009).

Por último, el control legal de depredadores a veces tiene efectos positivos en las poblaciones de otras presas no cinegéticas.
Por ejemplo, varios estudios han mostrado que el control de depredadores generalistas puede favorecer a algunas poblaciones de aves amenazadas (Côté y Sutherland 1997, Fletcher et al. 2010, Salo et al. 2010, Smith et al. 2010), siendo este efecto especialmente evidente en el caso de aves que crían en el suelo, como las aves esteparias o las limícolas. No obstante, esto no se ha observado en todos los casos, o en todas las especies estudiadas (ver, por ejemplo, Côté y Sutherland 1997, Fletcher et al. 2010). Como en el caso de la gestión de hábitat, existen pocos estudios sobre el efecto del control de depredadores en la fauna no cinegética en ambientes mediterráneos. Sin embargo, estos indican que, al menos en algunos casos, la abundancia o el éxito reproductor de ciertas especies son mayores en cotos donde se controlan depredadores generalistas. Por ejemplo, Suarez y colaboradores (1993) observaron que el éxito reproductor de los paseriformes que crían en el suelo era menor en zonas protegidas que en los cotos colindantes donde se controlaban zorros y perros asilvestrados. Estrada y colaboradores (datos sin publicar) observaron en un estudio realizado en el centro-sur de España que el control de depredadores estaba relacionado positivamente con la abundancia de avutarda (Otis tarda) y sisón (Tetrax tetrax), pero no con la de otras especies esteparias.

\section{Efectos de las sueltas de perdices de granja y gestión de cotos intensivos de perdiz}

Las sueltas de especies cinegéticas criadas en granja se han convertido en una práctica muy habitual durante las últimas décadas (Champagnon et al. 2012). En España, por ejemplo, se ha extendido de forma alarmante en las últimas décadas el uso de sueltas de perdices de granja o conejos (Blanco-Aguiar et al. 2008, Delibes-Mateos et al. 2008b). Estas sueltas se utilizan para suplementar poblaciones en declive, o como método para mantener la actividad cinegética en zonas donde las poblaciones naturales no son suficientes para mantener el nivel de extracción deseado. Además de sus efectos en las poblaciones nativas de las especies en cuestión (v.g. Casas et al. 2012, Díaz-Sánchez et al. 2012), las sueltas pueden tener efectos negativos en la fauna no cinegética, ya que pueden favorecer la introducción de parásitos nuevos en el medio, que podrían afectar negativamente a otras especies presentes en los mismos ecosistemas (v.g. Villanúa et al. 2007).

En España, existen cotos denominados "intensivos", caracterizados por la posibilidad legal de soltar perdices de granja sin límite numérico durante todo el año (incluyendo la temporada cinegética). En estos cotos se llegan a soltar decenas de miles de perdices al año y las prácticas de gestión son generalmente más intensivas que en otros tipos de cotos (Arroyo et al. 2012). Pero todavía hay poca información sobre el efecto que esta gestión intensiva, basada en la producción "artificial" de caza, pueda tener en la fauna de los cotos en los que se realiza. En un estudio reciente se ha observado que, a pesar de que las aves esteparias pueden verse favorecidas por ciertas medidas de gestión cinegética, la abundancia de las mismas era relativamente menor en cotos intensivos que en otro tipo de cotos (Estrada et al. datos sin publicar). Además, la riqueza de rapaces era también relativamente menor en cotos intensivos, donde en principio debería haber mayor disponibilidad de presas Estrada et al. datos sin publicar). Por tanto, las sueltas de perdices de granja u otras características de los cotos intensivos podrían estar afectando negativamente a estos dos grupos de aves.

\section{Efectos de las molestias asociadas a la caza en especies no cinegéticas}

Muchas especies reaccionan al ruido, a la presencia de humanos o de animales potencialmente peligrosos en las proximidades cambiando su comportamiento o el uso que hacen del espacio para evitarlos (González et al. 2006, Arroyo y Razin 2006). El desarrollo de la actividad cinegética puede, por tanto, producir por sí mismo efectos directos e indirectos en otras especies presentes, ya que está asociada a ruido (disparos) y a presencia de humanos y perros. 


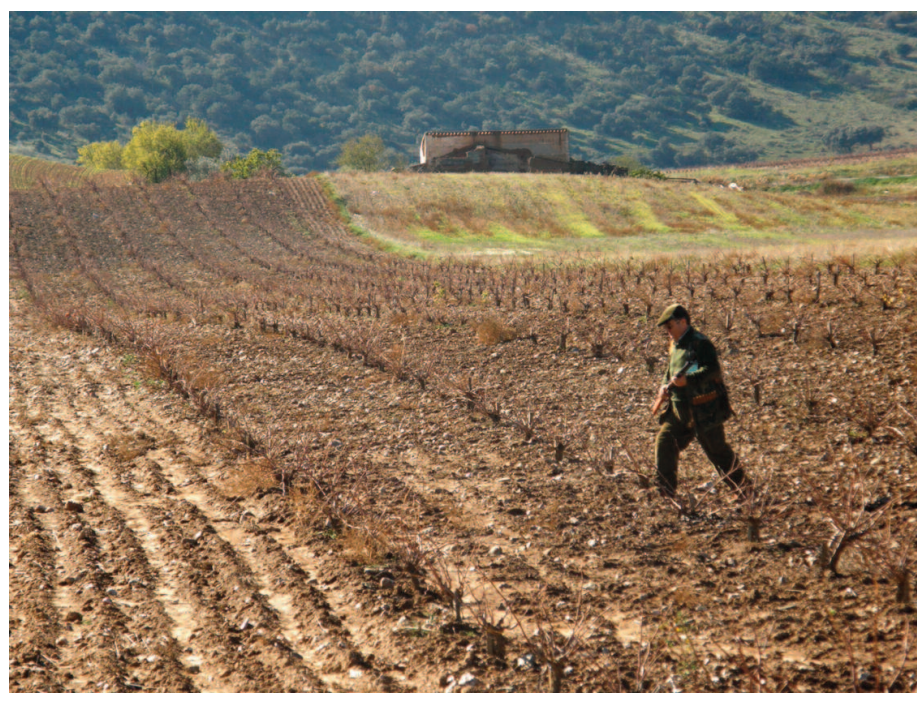

Figura 2. Cazador en plena jornada cinegética.

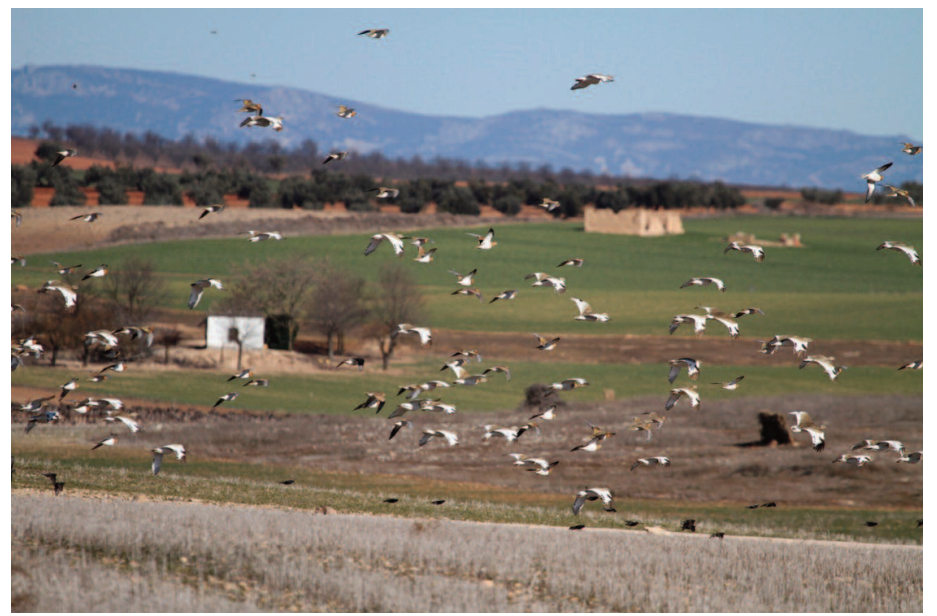

Figura 3. Bando mixto de sisones y gangas en coto cinegético de perdices.

Estos efectos han sido investigados sobre todo en zonas húmedas o hábitats forestales (v.g. Fox y Madsen 1997, Bregnballe et al. 2004, Duriez et al. 2005, Thiel et al. 2007), y también recientemente en medios agrícolas (Casas et al. 2009). Los diferentes estudios han mostrado que la caza causa molestias locales tanto a las especies cinegéticas como a otras especies protegidas. Las aves responden a las molestias provocadas por la caza cambiando su comportamiento, por ejemplo pasando más tiempo vigilando o volando que alimentándose o descansando, lo que puede tener efectos negativos en su condición física y por tanto en su supervivencia (Casas et al. 2009). En este sentido, un estudio reciente indica que el estrés fisiológico (medido a través de niveles de corticosterona, la hormona del estrés) aumenta durante los días de caza en el sisón común (R. Tarjuelo y M. Morales, com. pers.). Los animales también cambian su distribución habitual durante los días de caza, desplazándose a zonas donde no está permitida la caza para evitar molestias (Casas et al. 2009). En otro trabajo se ha observado que la abundancia de sisones durante el invierno era menor en zonas donde se encontraba una mayor densidad de cartuchos (BlancoAguiar et al. 2001), lo que sugiere que la presencia continua de molestias asociada a la caza podría ser negativa para esta especie.

En cualquier caso, este también es un tema relativamente poco estudiado en ambientes mediterráneos, aunque de gran importancia, y sería necesario estudiar mejor los efectos de la actividad cinegética sobre la fauna.

\section{Conclusiones}

Los estudios revisados en este trabajo muestran que existe un importante potencial para que la gestión cinegética favorezca a otras especies, con la excepción de los efectos negativos de ciertas prácticas sobre los depredadores protegidos. No obstante, parece que una gestión demasiado intensiva (sobre todo, cuando está basada fundamentalmente en la suelta de animales de granja) podría perjudicar a determinadas especies. Los beneficios de la gestión cinegética pueden ser particularmente marcados cuando dicha gestión está basada en el mantenimiento o creación de hábitats adecuados para las especies cinegéticas (mantenimiento de vegetación natural en la matriz agrícola, paisaje en mosaico o linderos), así como el aporte suplementario de agua y comida. El control legal de depredadores podría ser beneficioso para ciertas presas no cinegéticas, aunque este efecto positivo no se observa para todas las especies estudiadas, y esta herramienta de gestión está también asociada en ciertos casos a la práctica de métodos ilegales, lo que conlleva claros costes ambientales.

Globalmente, estos resultados indican que una opción óptima de gestión, que combine el mantenimiento de hábitats adecuados, un uso apropiado de bebederos y comederos, una caza basada en poblaciones naturales sostenibles ( $\sin$ suelta de animales de granja), y en algunos casos un control de depredadores legal y selectivo, podría beneficiar a otras especies de interés para la conservación. Por otro lado, para proteger tanto a las especies cinegéticas como a aquellas que conviven con estas es importante que la gestión incluya la regulación de la presión cinegética, y una mayor efectividad en el diseño de las reservas de caza. Sería particularmente interesante buscar formas para diferenciar a los cotos que realizan un tipo de gestión más respetuoso con el medio y sus especies, así como herramientas sociales o económicas que los apoyen.

\section{Referencias}

Amar, A., Court, I.R., Davison, M., Downing, S., Grimshaw, T., Pickford, T. 2012. Linking nest histories, remotely sensed land use data and wildlife crime records to explore the impact of grouse moor management on peregrine falcon populations. Biological Conservation 145:86-94.

Anderson, B.J., Arroyo, B., Collingham, Y.C., Etheridge, B., Fernandez-deSimon, J., Gillings, S., Gregory, R.D., Leckie, F.M., Sim, I.M.W., Thomas, C.D., Travis, J., Redpath, S.M. 2009. Using distribution models to test alternative hypotheses about a species' environmental limits and recovery prospects. Biological Conservation 142:488-499.

Angulo, E. 2003. Factores que afectan a la distribución y abundancia del conejo en Andalucía. Tesis doctoral, Universidad Complutense de Madrid, España.

Angulo, E., Villafuerte, R. 2003. Modelling hunting strategies for the conservation of wild rabbit populations. Biological Conservation 115:291-301.

Arroyo, B., Beja, P. 2002. Impact of hunting management practices on biodiversity. Report WP2 to REGHAB project. European Commission, Brussels. Disponible en: http://digital.csic.es/bitstream/10261/8260/1/WP2-repportf.pd

Arroyo, B., Razin, M. 2006. Effect of human activities on Bearded Vulture behaviour and breeding success in the French Pyrenees. Biological Conservation 128:276-284.

Arroyo, B., Delibes-Mateos, M., Díaz-Fernández, S., Viñuela, J. 2012. Hunting management in relation to profitability aims: red-legged partridge hunting in Central Spain. European Journal of Wildlife Research 58:847855

Beja, P., Gordinho, L., Reino, L., Loureiro, F., Santos-Reis, M., Borralho, R. 2009. Predator abundance in relation to small game management in southern Portugal: conservation implications. European Journal of Wildlife Research 55:227-238.

Blanco-Aguiar, JA, Virgós, E., Bautista LM y Viñuela, J. 2001. Análisis preliminar del estatus del sisón en castilla-la mancha y posibilidades de programas agroambientales de conservación. En: LPO (Ed). Actas del Cuarto Seminario Internacional sobre el sisón común, Castuera, España.

Blanco-Aguiar, J.A, González-Jara, P., Ferrero, M., Sánchez-Barbudo, I., Virgós, E., Villafuerte, R., Dávila, J.A. 2008. Assessment of restocking contributions to anthropogenic hybridization: The case of the Iberian red-legged partridge. Animal Conservation 11:535-545.

Blanco-Aguiar, J.A., Delibes-Mateos, M., Arroyo, B., Ferreras, P., Casas, F. Real, R., Vargas, J.M., Villafuerte, R., Viñuela, J. 2012. Is the interaction between rabbit hemorrhagic disease and hyperpredation by raptors a major cause of the red-legged partridge decline in Spain?. European Journal of Wildlife Research 58:433-439. 
Bregnballe, T., Madsen, J., Rasmussen, P.A.F. 2004. Effects of temporal and spatial hunting control in waterbirds reserves. Biological Conservation 119:93-104.

Buenestado, F.J., Ferreras, P., Blanco-Aguiar, J.A., Tortosa, F.S., Villafuerte R. 2009. Survival and causes of mortality among wild Red-legged Partridges Alectoris rufa in southern Spain: Implications for conservation. Ibis 151:720-730.

Caro, J., Ontiveros, D., Pizarro, M., Pleguezuelos, J.M. 2010. Habitat features of settlement areas used by floaters of Bonelli's and Golden eagles. Bird Conservation International 21:59-71.

Casas, F., Mougeot, F., Sánchez-Barbudo, I., Dávila, J.A., Viñuela, J. 2012. Fitness consequences of anthropogenic hybridization in wild red-legged partridge (Alectoris rufa, Phasianidae) populations. Biological Invasions 14:295-305.

Casas, F., Viñuela, J. 2010. Agricultural practices or game management: which is the key to improve red-legged partridge nesting success in agricultural landscapes? Environmental Conservation 37:177-186.

Casas, F., Mougeot, F., Viñuela, J., Bretagnolle, V. 2009. Effects of hunting on the behaviour and spatial distribution of farmland birds: importance of hunting-free refuges in agricultural areas. Animal Conservation 12:346-354.

Champagnon, J., Elmberg, J., Guillemain, M., Gauthier-Clerc, M., Lebreton J.D. 2012. Conspecifics can be aliens too: A review of effects of restocking practices in vertebrates. Journal for Nature Conservation 20:231 241.

Conover, R.R., Burger, L.W., Linder, E.T. 2007. Winter avian community and sparrow response to field border width. The Journal of Wildlife Management 71:1917-1923.

Côté, I.M., Sutherland, W.J. 1997. The effectiveness of removing predators to protect bird populations. Conservation Biology 11:395-405.

Delibes-Mateos, M., Gálvez-Bravo, L. 2009. El papel del conejo como especie clave multifuncional en el ecosistema mediterráneo de la Península Ibérica. Ecosistemas 18:14-25.

Delibes-Mateos, M., Redpath, S.M., Angulo, E., Ferreras, P., Villafuerte, R. 2007. Rabbits as a keystone species in southern Europe. Biological Conservation 137:149-156.

Delibes-Mateos, M., Ferreras, P., Villafuerte, R. 2008a. Rabbit populations and game management: the situation after 15 years of rabbit haemorrhagic disease in central-southern Spain. Biodiversity and Conservation 17:559-574.

Delibes-Mateos, M., Ramírez,, E., Ferreras, P., Villafuerte, R. 2008b. Translocations as a risk for the conservation of European wild rabbit Oryctolagus cuniculus lineages. Oryx 42:259-264.

Delibes-Mateos M., Ferreras P., Villafuerte R. 2009. Rabbit (Oryctolagus cuniculus) abundance and protected areas in central-southern Spain: why they do not match? European Journal of Wildlife Research 55:65-69.

Delibes-Mateos, M., Díaz-Fernández, S., Ferreras, P., Viñuela, J, Arroyo, B. 2013. The role of economic and social factors driving predator control in small game estates in central Spain. Ecology and Society 18:28.

Díaz-Fernández, S., Arroyo, B., Casas, F., Martinez-Haro, M., Viñuela, J. en prensa. Effect of game management on wild red-legged partridge abundance. PLOS One. (http://dx.plos.org/10.1371/journal.pone.0066671).

Díaz-Sánchez, S, Mateo Moriones, A., Casas, F., Höfle, U., 2012. Prevalence of Escherichia coli, Salmonella sp. and Campylobacter sp. in the intestinal flora of farm-reared, restocked and wild red-legged partridges (Alectoris rufa): is restocking using farm-reared birds a risk? European Journal of Wildlife Research 58:99-105.

Donald, P.F., Sanderson, F.J., Burfield, I.J., Van Bommel, F.P.J. 2006. Further evidence of continent-wide impacts of agricultural intensification on European farmland birds, 1990-2000. Agriculture, Ecosystems and Environment 116:189-196.

Duriez, O., Eraud, C., Barbraud, C., Ferrand, Y. 2005. Factors affecting population dynamics of Eurasian woodcocks wintering in France: assessing the efficiency of a hunting-free reserve. Biological Conservation 122:89-97.

Etheridge, B., Summers, R.W., Green, R.E. 1997. The effects of illegal killing and destruction of nests by humans on the population dynamics of the hen harrier Circus cyaneus in Scotland. Journal of Applied Ecology 34:1081-1105.

The Federation of Associations for Hunting and Conservation of the EU (FACE) 2005. Census of the number of hunters in Europe. http://www.faceeurope.org/fs-hunting.htm (updated 21.02.2005).
Ferreira, C., Touza, J., Rouco, C., Díaz-Ruíz, F., Fernández de Simón, J., Ríos-Saldaña, C., Ferreras, P., Villafuerte, R., Delibes-Mateos, M. En prensa. Habitat management as a generalized tool to boost European rabbits (Oryctolagus cuniculus) populations in the Iberian Peninsula: A cost-effectiveness analysis. Mammal Review. Doi:10.1111/mam.12006

Fletcher, K., Aebischer, N.J., Baines, D., Foster, R., Hoodless, A.N. 2010. Changes in breeding success and abundance of ground-nesting moorland birds in relation to the experimental deployment of legal predator control. Journal of Applied Ecology 47:263-272.

Fox, A.D., Madsen, J. 1997. Behavioural and distributional effects of hunting disturbance on waterbirds in Europe: implications for refuge design. Journal of Applied Ecology 34:1-13.

Gaudioso-Lacasa, V., Sánchez García-Abad, C., Prieto Martín, R., Bartolomé Rodríguez, D., Pérez Garrido, J., et al. 2010. Small game water troughs in a Spanish agrarian pseudo steppe: visits and water site choice by wild fauna. European Journal of Wildlife Research 56:591599.

Gonzalez, L.M., Arroyo, B., Margalida, A., Sánchez, R., Oria, J. 2006. Effect of human activities on the behaviour of breeding Spanish Imperial Eagles Aquila adalberti: management implications for the conservation of a threatened species. Animal Conservation 9:85-93.

González, L.M., Margalida, A., Mañosa, S., Sánchez, R., Oria, J., Molina, J.I., Caldera, J., Aranda, A., Prada, L. 2007. Causes and spatio-temporal variations of non-natural mortality in the Vulnerable Spanish imperial eagle Aquila adalberti during a recovery period. Oryx 41:495-502.

Halliday, T.R. 1980. The extinction of the passenger pigeon Ectopistes migratorius and its relevance to contemporary conservation. Biological Conservation 17:157-162

Hassall, M., Hawthorne, A., Maudsley, M., White, P., Cardwell, C. 1992. Effects of headland management on invertebrate communities in cereal fields. Agriculture, Ecosystems and Environment 40:155-178.

Henderson, I.G., Vickery, J.A., Carter, N. 2004. The use of winter bird crops by farmland birds in lowland England. Biological Conservation 118:21-32.

Hinsley, S.A., Bellamy, P.E. 2000. The influence of hedge structure, management and landscape context on the value of hedgerows to birds: a review. Journal of Environmental Management 60:33-49.

Leopold, B.D., Chamberlain, M. 2002. Predator control: here we go again. Proceedings of the annual conference of the Southeastern association of Fish and Wildlife Agencies 56:239-254.

Márquez, C., Vargas, J.M., Villafuerte, R., Fa, J.E. 2013. Risk mapping of illegal poisoning of avian and mamalian predators. Journal of Wildlife Management 77:75-83

Mateo-Tomás, P., Olea, P.P.,Sánchez-Barbudo, I.S., Mateo, R. 2012. Alleviating human-wildlife conflicts: Identifying the causes and mapping the risk of illegal poisoning of wild fauna. Journal of Applied Ecology 49:376-385.

Martínez, J., Viñuela, J., Villafuerte, R. 2002. Socio-economic aspects of gamebird hunting, hunting bags and assessment of the status of gamebird populations in REGHAB countries. Part 1: Socioeconomic and cultural aspects of gamebird hunting. Report on Workpackage 1 of the European Project REGHAB N EKV- 2000-00637.

Ministerio de Medio Ambiente y Medio Rural y Marino (MARM). 2006. http://www.magrama.gob.es/fr/biodiversidad/publicaciones/Anuario_Estadistica_2006_tcm12-219274.pdf

Myers, N., Mittermeier, R.A., Mittermeier, C.G., da Fonseca, G.A.B., Kent, J. 2000. Biodiversity hotspots for conservation priorities. Nature 403:853-858.

Oldfield, T.E.E., Smith, R.J., Harrop, S.R., Leader-Williams, N. 2003. Field sports and conservation in the United Kingdom. Nature 423:531-533.

Olivero, J., Márquez, A.L., Arroyo, B. 2011. Modelización de las áreas agrícolas y forestales de alto valor natural en España. Informe para el Ministerio de Medio Ambiente (MARM). Madrid. España. http://www.magrama.gob.es/es/biodiversidad/temas/ecosistemas-y-conectividad/Inf_final_mod_agrarias_forestales_a_v_n_espana_tcm7249125.pdf

Palomares, F., Delibes, M., Revilla, E., Calzada, J., Fedriani, J.M. 2001. Spatial ecology of Iberian lynx and abundance of European rabbits in southwestern Spain. Wildlife Monographs 148:1-36.

Parish, D.M.B., Sotherton, N.W. 2004. Game crops as summer habitat for farmland songbirds in Scotland. Agriculture, Ecosystems and Environment 104:429-438.

Reginfo, J.I. 2008. Un segmento del turismo internacional en auge: el turismo de caza. Cuadernos de Turismo 22:187-210. 
Ríos-Saldaña, C.A. 2010. Los planes técnicos de caza de Castilla-La Mancha y su aplicación en la gestión y conservación de las especies cinegéticas. Tesis doctoral, Universidad de Castilla-La Mancha, Ciudad Real, España.

Robertson, P.A. 1992. Woodland management for pheasants. Forestry Commission Bulletin 106. HMSO, London, UK.

Robertson, P.A., Park, K.J., Barton, A.F. 2001. Loss of heather Calluna vulgaris moorland in the Scottish uplands: the role of red grouse Lagopus lagpus scoticus management. Wildlife Biology 7:11-16.

Rodríguez, A., Delibes, M. 2004. Patterns and causes of non-natural mortality in the Iberian lynx during a 40 -year period of range contraction. Biological Conservation 118:151-161.

Sage, R.B., Parish, D.M.B., Woodburn, M.I.A., Thompson P.G.L. 2005 Songbirds using crops planted on farmland as cover for game birds. European Journal of Wildlife Research 51:248-253.

Salo, P., Banks, P.B., Dickman, C.R., Korpimäki, E. 2010. Predator manipulation experiments: impacts on populations of terrestrial vertebrate prey. Ecological Monographs 80:531-546.

Smart, J., Amar, A., Sim, I.M.W., Etheridge, B., Cameron, D., Christie, G., Wilson, J.D. 2010. Illegal killing slows population recovery of a re-introduced raptor of high conservation concern - The red kite Milvus milvus. Biological Conservation 143:1278-1286.

Smith, J., Potts, S.G., Woodcock, B.A., Eggleton, P. 2008. Can arable field margins be managed to enhance their biodiversity, conservation and functional value for soil macrofauna? Journal of Applied Ecology 45:269-278.

Smith, R.K., Pullin, A.S., Stewart, G.B., Sutherland W.J. 2010. Effectiveness of Predator Removal for Enhancing Bird Populations. Conservation Biology 24:820-829.

Stoate, C., Szczur, J. 2001. Could game management have a role in the conservation of farmland passerines? A case study from a Leicestershire farm. Bird Study 48:279-292.

Stoate, C., Szczur, J., Aebischer, N.J. 2003. Winter use of wild bird cover crops by passerines on farmland in northeast England. Bird study 50:15-21.

Suarez, F., Yanes, M., Herranz, J. 1993. Nature reserves and the conservation of Iberian shrubsteppe passerines: the paradox of nest predation. Biological Conservation 64:77-81.
Tapper, S.C. 2005. Nature's gain: how gamebird management has influenced wildlife conservation. The Game Conservancy Trust, Fordingbridge, Hampshire, UK.

Thiel D., Menoni E., Brenot J.F., Jenni, L. 2007. Effects of recreation and hunting on flushing distance of Capercaillie. Journal of Wildlife Management 71:1784-1792

Valkama, J., Korpimäki, E., Arroyo ,B., Beja, P., Bretagnolle, V., Bro, E., Kenward, R., Manosa, S., Redpath, S.M., Thirgood S., Vinuela, J. 2005. Birds of prey as limiting factors of gamebird populations in Europe: a review. Biological Reviews 80:171-203.

Villafuerte, R., Viñuela, J., Blanco, J.C. 1998. Extensive predator persecution caused by population crash in a game species: The case of red kites and rabbits in Spain. Biological Conservation 84:181-188.

Villanúa, D., Casas, F.,Viñuela, J., Gortázar, C., García de la Morena, E.L., Morales, M.B. 2007. First occurence of Eucoleus contortus in a little bustard Tetrax tetrax. A negative effect of red-legged partridge Alectoris rufa releases on steppe bird conservation? Ibis 149:405-406

Viñuela, J., Villafuerte, R. 2003. Predators and rabbits (Oryctolagus cunicuIus) in Spain: a key conflict for European raptor conservation. En: Thompson, DBA, Redpath, SM, Fielding AH, Marquiss, M., Galbraith CA (Eds.). Birds of prey in a changing environment, pp: 511-526. The Stationery Office, Edinburgh. UK.

Virgós, E., Travaini, A. 2005. Relationship between small-game hunting and carnivore diversity in central Spain. Biodiversity and Conservation 14:3475-3486.

Whitfield, D.P., McLeod, D.R.A., Watson, J., Fielding, A.H., Haworth, P.F. 2003. The association of grouse moor in Scotland with the illegal use of poisons to control predators. Biological Conservation 114:157-163.

Whitfield, D.P., Fielding, A.H., McLeod, D.R.A., Haworth, P.F. 2004. The effects of persecution on age of breeding and territory occupation in golden eagles in Scotland. Biological Conservation 118:249-259.

Yanes, M., Herranz, J., de la Puente, J., Suárez, F. 1998. La Perdiz Roja. Identidad de los depredadores e intensidad de la depredación. (coord M. Sáenz de Buruaga), pp. 135-147. La Perdiz Roja. I curso. Grupo Editorial V-FEDENCA. Madrid, España. 